\title{
LA ADAPTACIÓN DE LA FLORA Y LA FAUNA AL CAMBIO CLIMÁTICO EN UN PAISAJE FRAGMENTADO, Y EL DERECHO EUROPEO SOBRE LA CONSERVACIÓN DE LA NATURALEZA
}

\author{
Arie TROUWBORST* \\ Profesor de Derecho Internacional y Europeo, Tilburg Law School \\ Investigador, Tilburg Sustainability Center (TSC) \\ Profesor invitado, Universidad de Granada \\ a.trouwborst@uvt.nl
}

Recibido: 13 de octubre de 2011 / Aceptado: 29 de noviembre de 2010

RESUMEN: Este trabajo analiza el papel de la Directiva Aves y la Directiva Hábitats de la UE con relación a los corredores ecológicos (o conectividad ecológica) y teniendo en cuenta la fragmentación del paisaje y el cambio climático. Además de este análisis general, se centra en el caso de los Países Bajos, que resulta de especial interés.

RESUM: Aquest treball analitza el paper de les Directives d'Aus i Hàbitats de la UE amb relació als corredors ecològics (o connectivitat ecològica), tenint en compte la fragmentació del paisatge i el canvi climàtic. A més d'aquesta anàlisi general, se centra en el cas dels Països Baixos, que resulta d'interès especial.

\footnotetext{
* Este trabajo forma parte del proyecto "Towards Climate Change Proof International Nature Conservation Law: International Law and the Adaptation of Species and Ecosystems to Climate Change", financiado por la Organización Holandesa para la Investigación Científica (NWO) en el marco del "Innovational Research Incentives Scheme". El autor participa también en el proyecto "Legal Aspects of Connectivity Conservation", de la Unión Internacional para la Conservación de la Naturaleza (IUCN). El autor agradece las útiles sugerencias de mejora de Teresa Fajardo del Castillo y la ayuda de Elvira Martínez Camacho en la revisión del castellano.
} 
ABSTRACT: This study analyzes the role of the EU Birds and Habitats Directives regarding the establishment and maintenance of ecological corridors (or ecological connectivity), in light of the fragmentation of habitats and climate change. Special attention is paid to the Netherlands, whose practice is of particular interest in this context.

PALABRAS CLAVE: cambio climático — adaptación — fragmentación — corredores ecológicos — Directiva Aves y Directiva Hábitats

PARAULES CLAU: canvi climàtic — adaptació — fragmentació - connectivitat ecològica — Directiva d'aus i Directiva d'hàbitats.

KEYWORDS: Climate Change - Adaptation - Fragmentation - Ecological Connectivity — Birds and Habitats Directives

Sumario: I. Introducción. II. Fragmentación, cambio climático y corredores ecológicos. III. La Directiva Aves y la Directiva Hábitats y los corredores ecológicos. IV. Aplicación: el caso de los Países Bajos. V. Conclusiones. VI. Bibliografía.

\section{INTRODUCCIÓN}

En el ámbito de la protección del medio ambiente, la Unión Europea (UE) persigue unos objetivos muy ambiciosos. Una vez fijados en la legislación secundaria, principalmente en las directivas, estos objetivos brindan a los Estados miembros unos desafíos igual de ambiciosos. La historia enseña que frecuentemente los Estados miembros han errado en el cálculo de la libertad que, en mayor o menor medida, les corresponde a la hora de elegir los medios para alcanzar los objetivos prescritos por las directivas - y que son sistemáticamente acentuados por el Tribunal de Justicia de la UE- Notorios ejemplos de las directivas en cuestión son la Directiva Aves $^{1}$ y la Directiva Hábitats ${ }^{2}$, las cuales forman la normativa principal en el campo de la protección de la flora, la fauna y los hábitats silvestres en la UE.

\footnotetext{
${ }^{1}$ Directiva 2009/147, DOUE 2010, L 20/7 (versión codificada de la Directiva 79/409).

${ }^{2}$ Directiva 92/43, DOUE 1992, L 206/7.
} 
Un estudio reciente, financiado por el Gobierno holandés, habla de una práctica habitual por parte de los Estados miembros estudiados (Alemania, Países Bajos y Reino Unido) consistente en sobreestimar el grado de discreción nacional en la implementación de directivas en materia de medio ambiente ${ }^{3}$. Por supuesto, aumenta bastante el riesgo de incumplimiento cuando un Estado miembro opta deliberadamente por no ejercer su competencia para adoptar medidas nacionales de protección adicionales, es decir, por encima del mínimo prescrito por las directivas. Se observa una tendencia semejante, para seguir con el ejemplo de los Países Bajos, en las políticas de los últimos Gobiernos holandeses. El Acuerdo de Gobierno del gabinete instalado en 2010, presidido por el primer ministro Rutte, proclama decididamente que en el ámbito de la conservación de la naturaleza "los suplementos nacionales por encima de la normativa europea serán detectados y eliminados"4. Así, el mínimo europeo es convertido en el máximo nacional. El término común en el idioma holandés para referirse a los suplementos nacionales indicados es nationale koppen, que literalmente se traduciría como "cabezas nacionales" — que hay que cortar-. Sin embargo, para poder eliminar limpiamente estos suplementos nacionales, es fundamental saber con exactitud dónde termina el torso europeo y dónde empieza el cuello de la "cabeza nacional".

Y es ahí donde reside el problema —o, desde la perspectiva académica, lo que hace el tema atrayente- Es lo que se podría denominar el "área gris" de la Directiva Aves y la Directiva Hábitats. Está claro, por ejemplo, que los Estados miembros están obligados a designar zonas de especial protección (ZEP), las denominadas áreas Natura 2000. También está claro, por dar otro ejemplo, que están obligados a prohibir la captura y muerte de aves silvestres y de animales que pertenezcan a especies nombradas en el anexo IV de la Directiva Hábitats. No obstante, con respecto a otras medidas de conservación de la naturaleza, la existencia de obligaciones jurídicas resulta más bien debatible.

Se intenta en este trabajo contribuir a trazar el mapa de esa "área gris" de las directivas. En particular, se centra en los corredores ecológicos, que son aplicados para asegurar conectividad entre zonas naturales importantes. A su vez, el análisis de los corredores ecológicos en el marco de la Directiva Aves y la Directiva Hábitats se lleva a cabo a la luz de dos fenómenos que, sobre todo actuando en combinación, ejercen una creciente influencia

\footnotetext{
${ }^{3}$ DE BOER, S. et ál., De zoektocht naar nationale beleidsruimte in de EU-richtlijnen voor het milieu-en natuurbeleid. Een vergelijking van de implementatie van de Vogel- en Habitatrichtlijn, de Kaderrichtlijn Water en de Nitraatrichtlijn in Nederland, Engeland en Noordrijn-Westfalen, Wettelijke Onderzoekstaken Natuur en Milieu (WOt) werkdocument 174, Alterra, Wageningen, 2010.

${ }^{4}$ VVD-CDA Regeerakkoord "Vrijheid en Verantwoordelijkheid", 30 septiembre 2010, p. 14 (traducción por el autor).
} 
sobre la conservación de la flora y de la fauna silvestre. Estos dos fenómenos son la fragmentación del paisaje y el cambio climático. Después de llevar a cabo este análisis general, el artículo se centra en el caso de un Estado miembro, los Países Bajos, cuya práctica acerca de los corredores ecológicos resulta de especial interés.

\section{FRAGMENTACIÓN, CAMBIO CLIMÁTICO Y CORREDORES ECOLÓGICOS}

La fragmentación del paisaje se refiere a la conversión de grandes áreas de hábitat en áreas de hábitat más pequeñas y aisladas al dar a los hábitats naturales otros usos como la agricultura, la urbanización o la construcción de infraestructuras lineares como autovías y ferrocarriles. Un estudio publicado en septiembre de 2011 por la Agencia Europea de Medio Ambiente (EEA) y una agencia suiza midió por primera vez en detalle el grado de fragmentación del paisaje por infraestructuras de transporte y zonas urbanas en 28 países europeos ${ }^{5}$. El informe afirma que esta fragmentación tiene varios efectos ecológicos y contribuye de una forma apreciable al declive y a la pérdida de poblaciones de flora y fauna silvestre y a la amenaza de especies en Europa ${ }^{6}$. Para ilustrarlo, el informe cita la siguiente metáfora inventada por el escritor popular David Quammen:

"Let's start by imagining a fine Persian carpet and a hunting knife. The carpet is 12 feet by 18, say. That gives us 216 square feet of continuous woven material. We set about cutting the carpet into 36 equal pieces, each one a rectangle, two feet by three. [..] When we're finished cutting, we measure the individual pieces, total them up - and find that, lo, there's still nearly 216 square feet of recognisably carpetlike stuff. But what does it amount to? Have we got 36 nice Persian throw rugs? No. All we're left with is three dozen ragged fragments, each one worthless and commencing to come apart.

Now take the same logic outdoors and it begins to explain why the tiger, Panthera tigris, has disappeared from the island of Bali. It suggests why the jaguar, the puma, and 45 species of birds have been extirpated from a place called Barro Colorado Island - and why myriad other creatures are mysteriously absent from myriad other sites. An ecosystem is a tapestry of species and relationships. Chop away a section, isolate that section, and there arises the problem of unravelling"7.

\footnotetext{
5 EUROPEAN ENVIRONMENT AGENCY, SWISS FEDERAL OFFICE FOR THE ENVIRONMENT, Landscape fragmentation in Europe, Publications Office of the European Union, Luxembourg, 2011.

${ }^{6}$ Ibíd., p. 7.

${ }^{7}$ Citado del libro The song of the dodo (1996), en EUROPEAN ENVIRONMENT AGENCY, SWISS FEDERAL OFFICE FOR THE ENVIRONMENT, ibíd., p. 9.
} 
Muchas zonas altamente fragmentadas se hallan en Bélgica, los Países Bajos, Dinamarca, Alemania, Francia, Polonia y la República Checa. España es un país con un grado de fragmentación media, y sus tasas de fragmentación más altas se encuentran en la costa y alrededor de grandes ciudades. El informe subraya la urgencia de remediar el problema por medio de medidas de conectividad ecológica: "there is an urgent need for action".

Existe, en el ámbito de las ciencias naturales, una abundante literatura enfocada a los corredores ecológicos o la conectividad ecológica ${ }^{9}$. Una acreditada obra al respecto define así los corredores ecológicos:

"Ecological corridors are defined functionally to indicate connectivity and as physical structures to indicate connectedness. They are functional connections enabling dispersal and migration of species that could be subject to local extinction and they are landscape structures (other than core areas) varying in size and shape from wide to narrow and from meandering to straight, which represent links that permeate the landscape and maintain natural connectivity"10.

Los problemas ecológicos causados por la fragmentación del paisaje se ven aumentados de una forma considerable por el cambio climático. Una reacción natural de las poblaciones de plantas y animales ante un cambio de clima es un desplazamiento gradual, coincidiendo con el movimiento de las zonas climáticas, para no quedarse fuera de la zona climática que presenta las condiciones apropiadas para su supervivencia. El movimiento de estas zonas climáticas en Europa es generalmente hacia el norte y montaña arriba. Naturalmente, para que esta "dispersión climática" de la flora y fauna silvestre — que ya se ha puesto en marcha— se pueda desarrollar con éxito es imprescindible que los organismos puedan alcanzar los hábitats recién adecuados ${ }^{11}$. Este simple dato, sin embargo, no lo dice todo acerca del papel de la fragmentación y la conectividad ecológica en el marco de la adaptación de la naturaleza al cambio climático.

\footnotetext{
${ }^{8}$ Ibíd., p. 7.

${ }^{9}$ Se limita este trabajo a destacar dos: BENNETT, A.F., Linkages in the landscape: the role of corridors and

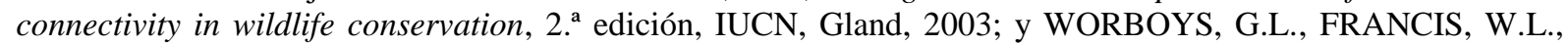
LOCKWOOD, M. (dir.), Connectivity conservation management: a global guide, Earthscan, Londres, 2010.

${ }^{10}$ WORBOYS et ál., ibíd., p. xxxii (referencias bibliográficas omitidas).

${ }^{11}$ Existe una literatura muy amplia sobre las consecuencias del cambio climático para la vida silvestre. Un intento para compilar y sintetizar esta literatura se puede encontrar en TROUWBORST, A., "International nature conservation law and the adaptation of biodiversity to climate change: a mismatch?", en Journal of Environmental Law, vol. 21, núm. 3, 2009, pp. 427-429, pp. 419-421.
} 
Una segunda, e importante, función de la conectividad ecológica en este marco es fortalecer las poblaciones para poder superar circunstancias extremas asociadas con el cambio climático. Se aclara esta función muy bien en un informe holandés de 2006:

"Condiciones meteorológicas extremas ocurrirán con más frecuencia. Sucesos extremos, como por ejemplo una primavera fría con mucha lluvia o largas temporadas de calor en verano, pueden ocasionar una elevada mortalidad o una fracasada reproducción. En consecuencia, los tamaños de las poblaciones muestran altibajos más pronunciados, y aumenta la probabilidad de extinción. La recuperación de una población después de un suceso semejante se produce con mucha más rapidez cuando la conectividad del hábitat es adecuada. En un paisaje fragmentado, en cambio, la recuperación tarda mucho más. Cuando aumenta la frecuencia de las alteraciones, esto puede causar la pérdida de especies" $"$.

Por lo tanto, existe un claro consenso en la literatura científica que afirma que es preciso el mantenimiento o restablecimiento de un grado adecuado de conectividad ecológica para facilitar la adaptación de la naturaleza al cambio climático ${ }^{13}$.

Aunque por motivos de espacio no pueda profundizar en ello, es conveniente mencionar que la importancia de los corredores ecológicos para la adaptación de las especies al cambio climático ha sido subrayada repetidas veces a nivel de la política internacional, por ejemplo en recomendaciones adoptadas por los Estados partes de diversos convenios mundiales y regionales en el campo de la conservación de la naturaleza ${ }^{14}$. Lo mismo ocurre con la política de la UE. Según el Libro Blanco de la Comisión Europea sobre la adaptación al cambio climático, "los impactos del cambio climático deben integrarse también en la gestión de la red Natura 2000 para garantizar la diversidad de espacios naturales y su interconexión y permitir que las especies puedan migrar y sobrevivir cuando cambian las condiciones climáticas"15. Recientemente, la Comisión y el Consejo han hecho un llamamiento al desarrollo de una “infraestructura verde" en el $83 \%$ del territorio de la UE que se sitúa fuera de la red Natura

\footnotetext{
${ }^{12}$ VOS, C.C., VAN DER VEEN, M., OPDAM, P.F.M., Natuur en klimaatverandering: wat kan het natuurbeleid doen?, Alterra, Wageningen, 2006, p. 7 (traducción del holandés por el autor).

${ }^{13}$ Ibíd.; véase también el esfuerzo de resumir esta literatura en TROUWBORST, "International nature conservation law..." cit., pp. 427-429.

${ }^{14}$ Véase TROUWBORST, ibíd.; y TROUWBORST, A., "Conserving European biodiversity in a changing climate: the Bern Convention, the EU Birds and Habitats Directives and the adaptation of nature to climate change', en Review of European Community and International Environmental Law, vol. 20, núm. 1, 2011, pp. 62-77.

${ }^{15}$ Libro Blanco "Adaptación al cambio climático: hacia un marco europeo de actuación”, Comunicación COM (2009) 147, 1 de abril de 2009, p. 13.
} 
2000, entre otras cosas para frenar la fragmentación de hábitats y facilitar la adaptación de la naturaleza al cambio climático ${ }^{16}$.

La principal cuestión tratada en este trabajo, sin embargo, es si además de una aspiración política, el establecimiento de una adecuada conectividad ecológica es también una obligación en el marco de la Directiva Aves y la Directiva Hábitats.

\section{LA DIRECTIVA AVES Y LA DIRECTIVA HÁBITATS Y LOS CORREDORES ECOLÓGICOS}

En la literatura académica jurídica se puede observar un creciente interés por la Directiva Aves y la Directiva Hábitats a la luz del cambio climático ${ }^{17}$. En este contexto, se ha declarado a menudo que el régimen de conservación de la naturaleza constituido por las directivas presenta puntos frágiles en cuanto a los corredores ecológicos ${ }^{18}$. En efecto, por una parte, los artículos 3 y 10 de la Directiva Hábitats, que tratan en términos específicos tales corredores, parecen carecer de "garras" 19 . Así, parecen dejar un amplio margen de decisión a los Estados miembros en cuanto a los corredores ecológicos:

"Cuando lo consideren necesario, los Estados miembros se esforzarán por mejorar la coherencia ecológica de Natura 2000 mediante el mantenimiento y, en su caso, el

\footnotetext{
${ }^{16}$ Se define la infraestructura verde como "una red interconectada de espacios naturales, incluidos algunos terrenos agrícolas, vías verdes, humedales, parques, reservas forestales y comunidades de plantas autóctonas, así como espacios marinos que regulan de forma natural los caudales de aguas pluviales, las temperaturas, el riesgo de inundaciones y la calidad del agua, el aire y los ecosistemas"; véase Comunicación COM (2010), 19 de enero de 2010, p. 6; Conclusiones del Consejo sobre Biodiversidad Post-2010, 15 de marzo de 2010, par. 6; y Comunicación COM (2011)244, 3 de mayo del 2011, p. 5.

${ }^{17}$ Véase, entre otros, WHEELER, K., "Bird protection and climate changes: a challenge for Natura 2000?", en Tilburg Foreign Law Review, vol. 13, núm. 3, 2006, pp. 283-299; WOLDENDORP, H.E., "Integratiedebat in het natuurbeschermingsbeleid", en Nederlands Juristenblad, núm. 45/46, 2007, pp. 2881-2886; CLIQUET, A., BACKES, C., HARRIS, J., HOWSAM, P., "Adaptation to climate change: legal challenges for protected areas", en Utrecht Law Review, vol. 5, núm. 1, 2009, pp. 158-175; ERENS, S., VERSCHUUREN, J., BASTMEIJER, K., "Adaptation to climate change to save biodiversity: lessons learned from African and European experiences", en Richardson, B.J. et ál. (dir.), Climate law and developing countries: legal and policy challenges for the world economy, Edward Elgar, Cheltenham, 2009, pp. 206-231; BASTMEIJER, C.J., WILLEMS, K., “'Robuust, verbonden en... beschermd.' Past een klimaatbestendig natuurbeleid met aandacht voor 'wilde natuur'-beleving in het juridische Natura 2000-jasje?", en Teesing, N. (dir.), Natuur(lijk) met recht beschermd: bouwstenen voor een effectieve en hanteerbare natuurbescherming, Boom Juridische uitgevers, La Haya, 2010, pp. 85-115; VERSCHUUREN, J., "Rethinking restoration in the European Union's Birds and Habitats Directives", en Ecological Restoration, vol. 28, núm. 4, 2010, pp. 431-439; TROUWBORST, "Conserving European biodiversity..." cit.

${ }^{18}$ Véase, entre otros, CLIQUET et ál., ibíd., p. 171; TROUWBORST, "International nature conservation law..." cit., pp. 439-440; ERENS et ál., ibíd., pp. 217-218; VERSCHUUREN, ibíd., p. 436.

${ }^{19}$ Verschuuren, ibíd.
} 
desarrollo de los elementos del paisaje que revistan primordial importancia para la fauna y la flora silvestre" 20 .

Se trata de aquellos elementos que, por su estructura lineal y continua (como los ríos con sus correspondientes riberas o los sistemas tradicionales de deslinde de los campos) o por su papel de puntos de enlace (como los estanques o los sotos), resultan esenciales para la migración, la distribución geográfica y el intercambio genético de las especies silvestres ${ }^{21}$.

Por otro lado, por específicos que sean los artículos 3 y 10, no son los únicos artículos de relevancia para el tema. Eso queda muy claro sobre todo teniendo en cuenta que, con respecto al cambio climático, los corredores ecológicos no solo sirven para hacer posible la dispersión de organismos silvestres, sino también para armar a las poblaciones para hacer frente a circunstancias extremas. De hecho, esa última función de los corredores ecológicos implica la relevancia de los artículos de la Directiva que, en principio, se orientan exclusivamente a la conservación y/o recuperación de las especies y los hábitats que ocurren dentro de las zonas Natura 2000, incluyendo los dos primeros apartados del conocido sexto artículo de la Directiva Hábitats.

Dada la mencionada función, el establecimiento y mantenimiento de una adecuada conectividad entre las zonas Natura 2000 deben considerarse obligatorios como consecuencia de la prescripción del artículo 6(1) de fijar "las medidas de conservación necesarias",22 que "respondan a las exigencias ecológicas de los tipos de hábitats naturales del Anexo I y de las especies del Anexo II presentes en los lugares”. Esta prescripción representa una obligación de resultado la cual, a su vez, debe ser interpretada a la luz del objetivo de la Directiva Hábitats de lograr "el mantenimiento o el restablecimiento, en un estado de conservación favorable, de los hábitats naturales y de las especies silvestres de la fauna y de la flora de interés comunitario"23.

\footnotetext{
${ }^{20}$ Habitatrichtlijn, art. 3(3); zie ook art. 10(1).

${ }^{21}$ Ibíd., art. 10(2).

${ }^{22}$ Énfasis añadido.

${ }^{23}$ Artículo 2(2) de la Directiva Hábitats. Según el artículo 1(e), aquello exige, en el caso de un hábitat, entre otras cosas que "la estructura y las funciones específicas necesarias para su mantenimiento a largo plazo existan y puedan seguir existiendo en un futuro previsible" (énfasis añadido). Según el artículo 1(i), el estado de conservación de una especie se considerará "favorable" cuando, entre otras cosas, los datos pertinentes indiquen que esta "sigue y puede seguir constituyendo a largo plazo un elemento vital de los hábitats naturales a los que pertenezca" (énfasis añadido). Véase también la guía producida por la Comisión Europea, Gestión de espacios Natura 2000: disposiciones del Artículo 6 de la Directiva 92/43/CEE sobre hábitats, 2000, p. 17.
} 
Similares conclusiones se derivan del artículo 6(2), el cual requiere que los Estados miembros tomen "las medidas apropiadas para evitar, en las zonas especiales de conservación, el deterioro de los hábitats naturales y de los hábitats de especies, así como las alteraciones [apreciables] que repercutan en las especies que hayan motivado la designación de las zonas". Esta disposición ha sido interpretada por el Tribunal de Justicia de la UE de manera constante como una obligación to do what it takes. Lo que es "apropiado" depende, en cada caso, del problema en cuestión, pero lo determinante es siempre el resultado ${ }^{24}$. De evidente importancia en el presente contexto es asimismo el carácter preventivo de la obligación, por la que se exige acción por parte de los Estados miembros antes de que se produzcan (más) impactos $\operatorname{adversos}^{25}$. Resulta rotundo, además, el hecho de que el ámbito del artículo 6(2) no se limita a medidas tomadas dentro de las zonas Natura 2000. En palabras de la Comisión Europea:

"Las medidas se refieren únicamente a especies y hábitats situados "en las zonas especiales de conservación". Sin embargo, puede ser necesario poner en práctica medidas fuera de las ZEC, por ejemplo, si hechos externos pueden tener un impacto sobre las especies y hábitats que se encuentran dentro de una ZEC. De hecho, el artículo no especifica que las medidas tengan que adoptarse en las ZEC, sino lo que se debe evitar en esos espacios" ${ }^{\prime 26}$.

Por ende, cuando queda patente que la existencia de conexiones adecuadas con otros espacios naturales es una condición previa para la supervivencia a largo plazo de ciertas especies o ciertos hábitats hábiles para ser clasificados como zonas Natura 2000, entonces la creación o el mantenimiento de dichas conexiones es necesario para poder cumplir el mandamiento del artículo 6(2). Consideraciones similares pertenecen a las obligaciones anticipatorias acerca de la protección genérica de las especies del anexo IV de la Directiva Hábitats ${ }^{27}$. En resumen, los Estados miembros deben atajar las causas de un estado de conservación no favorable, y eso requiere más que la mera defensa contra nuevos impactos adversos. Muchas veces es

\footnotetext{
${ }^{24}$ Para un ejemplo muy claro, véase TdJ 13 junio de 2002, asunto C-117/00, Comisión/Irlanda, Rec. 2002, p. I5335, punto 26-33.

${ }^{25}$ La redacción del artículo 6(2) pone en evidencia este carácter preventivo; véase también la guía de la Comisión Europea, Gestión de espacios ... cit., p. 24.

${ }^{26}$ Comisión Europea, ibíd. (énfasis como en original; ZEC se refiere a zonas de especial conservación).

27 Artículos 12(1) y 13(1) de la Directiva Hábitats; véase TdJ 11 enero de 2007, asunto C-183/05, Comisión/Irlanda, Rec. 2007, p. I-137, puntos 14, 18, 29-30. Para un análisis, véase TROUWBORST, "Conserving European biodiversity..." cit., p. 73; véase también TROUWBORST, A., DOTINGA, H.M., "Comparing European instruments for marine nature conservation: the OSPAR Convention, the Bern Convention, the Birds and Habitats Directives, and the added value of the Marine Strategy Framework Directive", en European Energy and Environmental Law Review, vol. 20, núm. 4, 2011, pp. 129-149, pp. 136137.
} 
necesario que los Estados miembros tomen la iniciativa implementando medidas básicas de mejora y restauración ${ }^{28}$. Como la Comisión Europea formulaba sucintamente en una carta dirigida recientemente a una ONG holandesa, relativa a especies y hábitats de interés comunitario, la Directiva Hábitats "requires taking all the measures necessary to achieve a favourable conservation status" ${ }^{29}$. Dicha interpretación y conclusiones coinciden con lo confirmado en un estudio sobre conectividad hecho para la Comisión Europea en $2007^{30}$. El estudio señala que, en principio, se deben tomar medidas para combatir la fragmentación "whenever they are necessary to maintain or restore FCS of habitats or species of Community interest" ${ }^{31}$.

Cabe afirmar, igualmente, que aunque los artículos 3(3) y 10 de la Directiva Hábitats contienen el discurso más específico relativo a los corredores ecológicos, ello de por sí no impide la aplicación de las obligaciones más rigurosas derivadas de los artículos 1, 2 y 6 de la misma Directiva. Una comparación con los artículos 12(1) y 12(4) de la Directiva Hábitats puede servir para ilustrarlo. El hecho de que en apariencia el artículo 12(4) haya sido redactado en particular para combatir, entre otras cosas, la captura incidental de tortugas marinas y cetáceos en redes de pesca no de por sí impide la aplicación a tales capturas de la prohibición genérica del artículo 12(1) de matar animales que pertenecen a especies del anexo $\mathrm{IV}^{32}$.

En dos casos españoles recientes, el Tribunal de Justicia ha destacado, en el marco de la Directiva Hábitats, la importancia de evitar la fragmentación de hábitats y de asegurar una adecuada conectividad ecológica. Una sentencia de 2010 acerca del lince ibérico (Lynx

\footnotetext{
${ }^{28}$ Véase, por ejemplo, VERSCHUUREN, "Rethinking restoration..." cit.; o BACKES, C.W., POORTINGA, M.A., WOLDENDORP, H.E., "Natuurbescherming in de Natuurwet: kop eraf?", en Teesing, N. (dir.), Natuur(lijk) met recht beschermd: bouwstenen voor een effectieve en hanteerbare natuurbescherming, Boom Juridische uitgevers, La Haya, 2010, pp. 17-57, p. 29.

${ }^{29}$ Carta de la Comisión Europea a Natuurmonumenten del 9 de diciembre de 2010, sobre el área Natura 2000 Haringvliet, disponible en: http://www.natuurmonumenten.nl/content/europese-commissie-intrekkingkierbesluit-onacceptabel.

${ }^{30}$ KETTUNEN, M. et ál., Guidance on the maintenance of landscape connectivity features of major importance for wild flora and fauna: guidance on the implementation of Article 3 of the Birds Directive (79/409/EEC) and Article 10 of the Habitats Directive (92/43/EEC), Institute for European Environmental Policy, Bruselas, 2007.

${ }^{31}$ Ibíd., p. 7 ("FCS" significa "favourable conservation status"). A propósito, el documento afirma en general que sus conclusiones no necesariamente representan la opinión de la Comisión.

${ }^{32}$ Véase, por ejemplo, la respuesta del 11 de febrero de 2008 del comisario Dimas a la pregunta parlamentaria E5890/07 sobre la captura accidental de marsopas comunes (Phocoena phocoena) en redes de enmalle, en la que el comisario declara que esta captura podría violar el artículo 12(1) y el artículo 12(4). Véase también TROUWBORST, A., DOTINGA, H.M., "Soortenbescherming in de Noordzee: laveren door een warnet aan internationale, Europese en Nederlandse regels met de bruinvis als loods", Tijdschrift voor Omgevingsrecht, núm. 3, 2008, pp. 90-101, p. 94.
} 
pardinus) en Doñana afirma que "las infraestructuras lineales de trasporte pueden constituir una auténtica barrera para algunas especies contempladas en la Directiva hábitats y, al fragmentar de ese modo su zona de expansión natural, favorecer la endogamia y la deriva genética de dichas especies"33. La otra sentencia, que fue pronunciada en noviembre de 2011 , concierne al efecto fragmentador de unas minas de carbón a cielo abierto en la zona Natura 2000 del Alto Sil en Castilla y León ${ }^{34}$. Destaca el impacto de estas minas sobre un corredor ecológico de gran valor para el oso pardo (Ursus arctos), el denominado corredor de Leitariegos. Este corredor conecta el hábitat del oso en Castilla y León con zonas oseras en Asturias y constituye, según describe la sentencia, "una vía de paso de gran importancia para la población occidental de la citada especie, que permite, en particular, la comunicación entre dos núcleos de reproducción muy importantes” ${ }^{\text {} 35}$. El Tribunal cita un informe ambiental que afirma "que los osos se alejan entre 3,5 y $5 \mathrm{~km}$ de las zonas de impacto de los ruidos y vibraciones ocasionados por las explotaciones mineras, y que aquella explotación impedirá o dificultará en mucha mayor medida el acceso del oso pardo a dicho corredor" ${ }^{\text {"36. Tales }}$ impactos son calificados por el Tribunal como perturbaciones de la zona Natura 2000 del Alto Sil "que resultan significativas desde el punto de vista de la conservación del oso pardo", y, por consiguiente, suponen un incumplimiento del artículo 6(2) de la Directiva Hábitats ${ }^{37}$. El Tribunal llega a similares conclusiones con respecto al urogallo (Tetrao urogallus), considerando que varias explotaciones mineras en la zona "pueden producir un 'efecto barrera' que contribuya a la fragmentación del hábitat del urogallo y al aislamiento de determinadas subpoblaciones de esta especie” ${ }^{, 38}$. Cabe observar que las minas se encuentran dentro de la zona Natura 2000 en cuestión, y que los corredores ecológicos afectados también están situados dentro de esta zona y de zonas Natura 2000 adyacentes. No obstante, es probable que el Tribunal hubiera llegado a idénticas conclusiones en un caso imaginario con poblaciones de osos o urogallos en dos zonas no adyacentes Natura 2000 conectadas por un corredor sin aquel estatus jurídico. En tal caso, el artículo 6(2) exigiría que el corredor, aunque situado fuera de las zonas Natura 2000, mantuviera su función para garantizar la conservación de las poblaciones de osos o urogallos dentro de esas zonas.

\footnotetext{
${ }^{33}$ TdJ 20 mayo de 2010, asunto C-308/08, Comisión/España, Rec. 2010, p. I-4281, punto 25.

${ }^{34}$ TdJ 24 noviembre de 2011, asunto C-404/09, Comisión/España, todavía no publicada.

${ }^{35}$ Ibíd., punto 189.

${ }^{36}$ Ibíd., punto 188.

${ }^{37}$ Ibíd., punto 191.

${ }^{38}$ Ibíd., punto 148.
} 
También parecen relevantes en el presente contexto los artículos 3 y 4 de la Directiva Aves. Conviene recordar en este contexto que, a pesar de su capacidad de volar, varias especies de aves son voladoras de corta distancia y están muy vinculadas a sus hábitats específicos, y, por lo tanto, son vulnerables ante la fragmentación de su entorno. Ejemplos son el ya mencionado urogallo y su pariente el gallo lira (Tetrao tetrix). El artículo 3 obliga a los Estados miembros a tomar, para cada una de las especies de aves silvestres, "todas las medidas necesarias para preservar, mantener o restablecer una diversidad y una superficie suficiente de hábitats"39. Entre las medidas requeridas se encuentra el "mantenimiento y ordenación de acuerdo con los imperativos ecológicos de los hábitats que se encuentren en el interior y en el exterior de las zonas de protección" ${ }^{40}$. Considerando que el artículo 3 se aplica también (o mejor dicho, sobre todo) fuera de las zonas Natura 2000, exigiría medidas contra la fragmentación, incluso si la única función de los corredores ecológicos fuera facilitar la dispersión de organismos inducida por el cambio climático. Igual que los primeros dos apartados del artículo 6 de la Directiva Hábitats, el artículo 3 parece haber sido redactado como una obligación anticipatoria y de resultado, y parece ser entendido como tal por el Tribunal de Justicia ${ }^{41}$. La constatación de que, según las circunstancias, garantizar la conectividad ecológica puede constituir una condición para el cumplimiento de la Directiva Aves de nuevo encaja con lo sugerido al respecto en el estudio de 2007 mencionado anteriormente ${ }^{42}$.

El anterior análisis no ha eliminado todavía todos los interrogantes acerca del dónde, el cuándo y otros detalles respecto a las obligaciones de crear y proteger corredores ecológicos. Las obligaciones en cuestión no solo dependen del contexto en cada caso, sino que también están relacionadas con incertidumbres importantes en cuanto a las consecuencias exactas del cambio climático para las especies y los hábitats. Esto introduce en las reglas del juego el conocido principio de precaución. Este principio constituye uno de los pilares de la política de la UE en el campo del medio ambiente ${ }^{43}$, "a la luz del cual debe interpretarse la Directiva sobre los hábitats" ${ }^{44}, \mathrm{y}$, sin duda, también la Directiva Aves. La esencia del principio de precaución, según lo interprete el Tribunal de Justicia en el ámbito de la conservación de

\footnotetext{
${ }^{39}$ Artículo 3(1) de la Directiva Aves.

${ }^{40}$ Artículo 3(2)(b).

${ }^{41}$ Asunto C-117/00, cit., puntos 15 y 21.

${ }^{42}$ KETTUNEN et ál., Guidance on the maintenance... cit., p. 7: "connectivity measures should be implemented whenever they are required to maintain populations in accordance with Article 2 of the [Birds] directive".

${ }^{43}$ Tratado de Funcionamiento de la Unión Europea, artículo 191(2).

${ }^{44}$ TdJ 7 septiembre de 2004, asunto C-127/02, Kokkelvisserij, Rec. 2004, p. I-7405, punto 44.
} 
naturaleza, es in dubio pro natura ${ }^{45}$. Como hemos afirmado antes, la literatura científica demuestra que, en general, para muchas especies la existencia de conexiones adecuadas entre las zonas donde habitan será fundamental para alcanzar un estado de conservación favorable a largo plazo. Al mismo tiempo, resulta difícil pronosticar con exactitud para qué poblaciones, zonas y momentos, y en qué medida, este será el caso. Dadas estas circunstancias, una interpretación de los artículos antes tratados de las directivas que tenga en cuenta el principio de precaución parece indicar una obligación para los Estados miembros de crear, de una manera preventiva, una amplia infraestructura ecológica que sea adecuada para todos los grupos de especies. Es decir, los Estados miembros no deberían limitar la toma de medidas de conexión a los casos en que las investigaciones científicas demuestren de una forma concluyente que alguna especie ya se encuentra en dificultades a consecuencia de la fragmentación de su hábitat. Además, esta proposición se ve apoyada por un reciente análisis de obligaciones relevantes en el Convenio de Berna Relativo a la Conservación de la Vida Silvestre y del Medio Natural en Europa ${ }^{46}$.

\section{APLICACIÓN: EL CASO DE LOS PAÍSES BAJOS}

Con su denominada Estructura Ecológica Principal (Ecologische Hoofdstructuur o EHS), hasta hace poco los Países Bajos estaban entre los líderes respecto al tema de las redes y los corredores ecológicos en Europa. La creación de esta infraestructura ecológica a nivel nacional empezó en 1990 con el objetivo de remediar la fragmentación de la naturaleza holandesa $^{47}$. Las áreas Natura 2000 forman parte integrante de la EHS. En el año 2000, cuando se hizo evidente que el diseño original de la EHS no iba a ser suficiente para lograr la calidad ecológica deseada — con hábitats nuevos demasiado separados, corredores ecológicos demasiado pequeños y demasiados obstáculos formados por infraestructuras no ecológicas (carreteras, etc.)—, se añadieron al plan unas denominadas "zonas conectoras robustas"

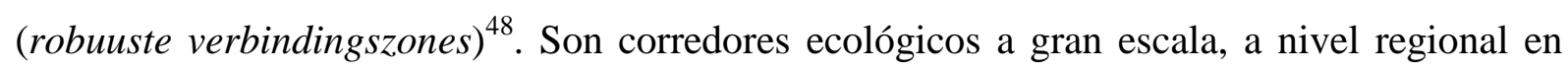

\footnotetext{
${ }^{45}$ Ibíd.

${ }^{46}$ Convenio Relativo a la Conservación de la Vida Silvestre y del Medio Natural en Europa, 19 de septiembre de 1979, ETS núm. 104. Véase para el análisis TROUWBORST, "Conserving European biodiversity...” cit., trabajo que ha sido distribuido también como Doc. T-PVS/Inf(2011)21 del Convenio de Berna.

${ }^{47}$ Ministerie van LNV, Natuurbeleidsplan, 1990.

48 Ministerie van LNV, Nota "Natuur voor mensen, mensen voor natuur", 2000; véase también BROEKMEYER, M., STEINGRÖVER, E., Handboek robuuste verbindingen, Alterra, Wageningen, 2001.
} 
vez de local. Durante las dos décadas pasadas, la EHS ha ido creciendo paso a paso, aunque con algunos retrasos. Su finalización estaba prevista para el 2018.

Pese a ello, en 2010 el gabinete tomó la polémica decisión de realizar la red de un modo bastante más reducido, para rebajar los gastos públicos. Decidió no dedicar más fondos a la adquisición de terreno y cancelar por completo las "zonas conectoras robustas" 49 . En base a esto, el Gobierno parte claramente de la premisa de que estas zonas conectoras, igual que las demás partes de la Estructura Ecológica Principal que no se califican como zonas Natura 2000, tan solo constituyen suplementos nacionales que complementan la normativa europea (“cabezas nacionales") y pueden ser eliminados sin violar la Directiva Aves ni la Directiva Hábitats. El análisis de las directivas en la sección previa de este trabajo, sin embargo, parece poner en duda esta premisa e indicar que la situación jurídica depende en gran medida de las circunstancias concretas. Por lo tanto, vale la pena examinar con un poco más de detalle el caso holandés.

En un estudio de 2010, el Planbureau voor de Leefomgeving (un organismo asesor que aconseja al Gobierno holandés en materia medioambiental) comprobó que aún no se había frenado el empeoramiento de la naturaleza en los Países Bajos, ya que "las poblaciones de especies vulnerables y la calidad de los ecosistemas vulnerables están en proceso de deterioro" ${ }^{50}$. En los Países Bajos, la gran mayoría de las especies y los hábitats de interés comunitario se encuentran en un estado de conservación desfavorable o muy desfavorable, y las cifras holandesas, además, son bajas en comparación con otros Estados miembros ${ }^{51}$.

Dado el elevado grado de fragmentación de la naturaleza holandesa y el previsible impacto del cambio climático, no cabe duda de la importancia de la Estructura Ecológica Principal en general y de las "zonas conectoras robustas" en particular. En este contexto, otro informe antes citado recomienda justamente "una implementación acelerada de las zonas conectoras robustas" y el establecimiento de "una buena coherencia entre las zonas Natura 2000 y las otras zonas naturales"52. El papel fundamental de las zonas conectoras robustas en la adaptación de la flora y la fauna al cambio climático se ha confirmado en un estudio centrado

\footnotetext{
${ }^{49}$ VVD-CDA Regeerakkoord... cit., p. 13.

${ }^{50}$ M.P. VAN VEEN et ál., Evaluatie biodiversiteitsdoelstelling 2010: achtergronddocument bij de Balans van de Leefomgeving 2010, Planbureau voor de Leefomgeving, La Haya/Bilthoven, 2010, p. 7 (traducción del holandés por el autor).

${ }^{51}$ Ibíd., p. 20, 24 en 25; véase también Comunicación COM (2009) 358, 13 de julio de 2009.

${ }^{52}$ VOS et ál., Natuur en klimaatverandering... cit., p. 3 (traducción del holandés por el autor).
} 
específicamente en la relación entre ambas y financiado por el Gobierno holandés ${ }^{53}$ : "La fragmentación de hábitats puede causar obstáculos, o embotellamientos, cuando especies se ven forzadas a desplazarse siguiendo el movimiento de las zonas climáticas" ${ }^{\text {"54 }}$. Concluye el estudio que "las zonas conectoras robustas contribuyen de una manera significativa a resolver los embotellamientos provocados por el cambio climático" ${ }^{\text {} 55}$.

Por último, en su reacción al Libro Blanco de la Comisión Europea sobre la adaptación al cambio climático, el gabinete holandés anterior declaró que compartía la aspiración de conseguir una "red de zonas naturales resistente al cambio climático" ${ }^{56}$. El Gobierno expresó lo siguiente:

"De momento no existe una red robusta de zonas naturales en Europa, a pesar de que el artículo 10 de la Directiva Hábitats tiene como objetivo garantizar una adecuada conectividad dentro de la red Natura 2000. A causa del cambio climático, la necesidad de lograr esto es ahora mayor que nunca", ${ }^{, 57}$.

Según la misma declaración, una red semejante debería incluir zonas conectoras robustas, incluyendo corredores ecológicos internacionales ${ }^{58}$.

Por consiguiente, todo lo susodicho parece confirmar la opinión de algunos catedráticos, que en 2010 dijeron en un periódico holandés que la política del Gobierno actual acerca de la EHS resulta "indefendible desde un punto de vista jurídico"59.

\section{CONCLUSIONES}

Evidentemente, un término clave en este trabajo es la obligación de resultado, más concretamente en el marco del deber de los Estados miembros de asegurar un "estado de conservación favorable" para ciertas especies y hábitats. Sin embargo, comparado, por

53 GEERTSEMA, W. et ál., Robuuste verbindingen en klimaatverandering, Alterra-rapport 1886, Alterra, Wageningen, 2009.

${ }^{54}$ Ibíd., p. 9 (traducción por el autor).

${ }^{55}$ Ibíd., p. 11.

${ }^{56}$ Declaración del Gobierno del 23 de abril de 2009, en Kamerstukken II, 2008-2009, 22 112, nr. 865, p. 7 (traducción por el autor).

${ }^{57}$ Ibíd.

${ }^{58}$ Ibíd.

${ }^{59}$ C. Backes, K. Bastmeijer y J. Verschuuren (y también firmados por A. Freriks, R. Gerlagh, P. Opdam, J. Schaminée, R. Uylenburg \& A. de Zeeuw), "Goed natuurbeleid is ook economisch van belang”, en el periódico Trouw del 5 de noviembre de 2010; véase también BACKES et ál., "Natuurbescherming in de Natuurwet..." cit., p. 29-30. 
ejemplo, con las obligaciones de respetar ciertas concentraciones máximas de determinados contaminantes en el aire después de una fecha prefijada, no resulta tan fácil establecer cuándo dicho deber ha sido cumplido y cuándo no. Es decir, en primer lugar, la Directiva Aves y la Directiva Hábitats no contienen una fecha límite al respecto. En segundo lugar, medir un estado de conservación es más difícil que medir una concentración de una sustancia química en el aire. En tercer lugar, en general es complicado determinar qué medidas prácticas hacen falta para lograr objetivos ecológicos. Dadas estas circunstancias y la interpretación estricta y teleológica de la Directiva Aves y la Directiva Hábitats por parte del Tribunal de Justicia, resulta arriesgado para los Estados miembros apostar bajo.

El tema de los corredores ecológicos ilustra esta observación general de una forma patente. Por ejemplo, con la anulación de las zonas conectoras robustas los Países Bajos se adentran manifiestamente en la zona de peligro, ya que está claro que el mantenimiento de una adecuada conectividad ecológica no puede ser descartado sin más como una medida optativa nacional. Es más, como se señaló arriba, del impacto combinado de la fragmentación del paisaje y el cambio climático parece derivarse la obligación de que los Estados miembros creen, de forma anticipatoria, una amplia infraestructura ecológica que sea adecuada para todos los grupos de especies.

Por otro lado, no se debería olvidar que al fin y al cabo sigue tratándose de obligaciones de resultado. Es decir, volviendo al caso de los Países Bajos, si el Gobierno holandés lograra demostrar que es capaz de alcanzar un estado de conservación favorable para las especies y los hábitats implicados sin zonas conectoras robustas, no habría problema. No obstante, a la luz de los conocimientos actuales esta posibilidad resulta algo hipotética.

De cualquier modo, es previsible que, a medida que avancen el cambio climático y la fragmentación del paisaje, el tema de la conectividad ecológica jugará un papel cada vez más importante en el marco del derecho de la conservación de la naturaleza.

\section{BIBLIOGRAFÍA}

BACKES, C.W., POORTINGA, M.A., WOLDENDORP, H.E., "Natuurbescherming in de Natuurwet: kop eraf?", en Teesing, N. (dir.), Natuur(lijk) met recht beschermd: bouwstenen voor een effectieve en hanteerbare natuurbescherming, Boom Juridische uitgevers, La Haya, 2010, pp. 17-57, p. 29. 
BASTMEIJER, C.J., WILLEMS, K., “'Robuust, verbonden en... beschermd.' Past een klimaatbestendig natuurbeleid met aandacht voor 'wilde natuur'-beleving in het juridische Natura 2000-jasje?”, en Teesing, N. (dir.), Natuur(lijk) met recht beschermd: bouwstenen voor een effectieve en hanteerbare natuurbescherming, Boom Juridische uitgevers, La Haya, 2010, pp. 85-115.

BENNETT, A.F., Linkages in the landscape: the role of corridors and connectivity in wildlife conservation, 2. a edición, IUCN, Gland, 2003.

BROEKMEYER, M., STEINGRÖVER, E., Handboek robuuste verbindingen, Alterra, Wageningen, 2001.

CLIQUET, A., BACKES, C., HARRIS, J., HOWSAM, P., "Adaptation to climate change: legal challenges for protected areas", en Utrecht Law Review, vol. 5, núm. 1, 2009, pp. 158175.

DE BOER, S. et ál., De zoektocht naar nationale beleidsruimte in de EU-richtlijnen voor het milieu- en natuurbeleid. Een vergelijking van de implementatie van de Vogel- en Habitatrichtlijn, de Kaderrichtlijn Water en de Nitraatrichtlijn in Nederland, Engeland en Noordrijn-Westfalen, Wettelijke Onderzoekstaken Natuur en Milieu (WOt) werkdocument 174, Alterra, Wageningen, 2010.

ERENS, S., VERSCHUUREN, J., BASTMEIJER, K., “Adaptation to climate change to save biodiversity: lessons learned from African and European experiences", en Richardson, B.J. et ál. (dir.), Climate law and developing countries: legal and policy challenges for the world economy, Edward Elgar, Cheltenham, 2009, pp. 206-231.

EUROPEAN ENVIRONMENT AGENCY/SWISS FEDERAL OFFICE FOR THE ENVIRONMENT, Landscape fragmentation in Europe, Publications Office of the European Union, Luxemburgo, 2011.

GEERTSEMA, W. et ál., Robuuste verbindingen en klimaatverandering, Alterra-rapport 1886, Alterra, Wageningen, 2009.

KETTUNEN, M. et ál., Guidance on the maintenance of landscape connectivity features of major importance for wild flora and fauna: guidance on the implementation of Article 3 of the Birds Directive (79/409/EEC) and Article 10 of the Habitats Directive (92/43/EEC), Institute for European Environmental Policy, Bruselas, 2007. 
TROUWBORST, A., "International nature conservation law and the adaptation of biodiversity to climate change: a mismatch?”, en Journal of Environmental Law, vol. 21, núm. 3, 2009, pp. 427-429.

— "Conserving European biodiversity in a changing climate: the Bern Convention, the EU Birds and Habitats Directives and the adaptation of nature to climate change", en Review of European Community and International Environmental Law, vol. 20, núm. 1, 2011, pp. 6277.

TROUWBORST, A., DOTINGA, H.M., "Soortenbescherming in de Noordzee: laveren door een warnet aan internationale, Europese en Nederlandse regels met de bruinvis als loods", Tijdschrift voor Omgevingsrecht, núm. 3, 2008, pp. 90-101.

- "Comparing European instruments for marine nature conservation: the OSPAR Convention, the Bern Convention, the Birds and Habitats Directives, and the added value of the Marine Strategy Framework Directive”, en European Energy and Environmental Law Review, vol. 20, núm. 4, 2011, pp. 129-149.

VAN VEEN, M.P. et ál., Evaluatie biodiversiteitsdoelstelling 2010: achtergronddocument bij de Balans van de Leefomgeving 2010, Planbureau voor de Leefomgeving, La Haya/Bilthoven, 2010.

VERSCHUUREN, J., "Rethinking restoration in the European Union's Birds and Habitats Directives”, en Ecological Restoration, vol. 28, núm. 4, 2010, pp. 431-439.

VOS, C.C., VAN DER VEEN, M., OPDAM, P.F.M., Natuur en klimaatverandering: wat kan het natuurbeleid doen?, Alterra, Wageningen, 2006.

WHEELER, K., “Bird protection and climate changes: a challenge for Natura 2000?", en Tilburg Foreign Law Review, vol. 13, núm. 3, 2006, pp. 283-299.

WOLDENDORP, H.E., "Integratiedebat in het natuurbeschermingsbeleid", en Nederlands Juristenblad, núm. 45/46, 2007, pp. 2881-2886.

WORBOYS, G.L./FRANCIS, W.L./LOCKWOOD, M. (dir.), Connectivity conservation management: a global guide, Earthscan, Londres, 2010. 